\section{Ciência e Tecnologia na Sociedade Digital: Visão de alunos do Curso de Licenciatura em Química.}

UTE. Revista de Ciències de l'Educació

2018 núm. 2. Pàg. 52-61

ISSN 1135-1438. EISSN 2385-4731

http://revistes.publicacionsurv.cat/index.php/ute

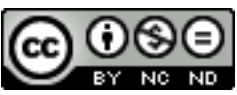

DOI: https://doi.org/10.17345/ute.2018.2.3539

\title{
Resumo
}

O trabalho foi realizado com os estudantes do Curso de Química da Universidade Estadual do Ceará, na disciplina de Ciência, Tecnologia e Sociedade. Foram entrevistados dezesseis alunos. Eles responderam a um questionário elaborado pelo Projeto Iberoamericano de Evaluación de Actitudes Relacionadas con la Ciencia, la Tecnología y la Sociedad (PIEARCTS). Este projeto foi aplicado em países da América do Sul como Brasil, Colômbia, Argentina e Venezuela; como também Portugal e Espanha. Os resultados das questões F1-20141 e F1-20411 foram semelhantes aos obtidos em pesquisa realizada no Centro Federal de Educação Tecnológica Celso Suckow da Fonseca - Rio de Janeiro em dezembro de 2010. Além dessas questões utilizou-se, do referido questionário, as questões F1-40161, F1-40221 e F1-40531 que tratam da Influência da Ciência e Tecnologia na Sociedade. Os resultados mostraram que após as intervenções didáticas, os respondentes se mostraram atualizados com os efeitos da tecnologia no mundo social e acreditam que o avanço tecnológico pode melhorar a qualidade de vida, quando utilizados com discernimento.

Palavras-chaves: Ciência. Tecnologia. Educação.

\begin{abstract}
This work was carried out with the students of the Chemistry Course of the State University of Ceara, in the course of Science, Technology and Society. Sixteen students were interviewed and they answered a questionnaire prepared by the Iberoamerican Project for the Evaluation of Attitudes Related to Science, Technology and Society (PIEARCTS). This project was applied in South American countries such as Brazil, Colombia, Argentina and Venezuela; as well as Portugal and Spain. The results of questions F1-20141 and F1-20411 were similar to those obtained in a survey conducted at Federal Center of Technological Education - Rio de Janeiro in December 2010. In addition to these questions, questions F1-40161, F140221 and F1-40531 dealing with the Influence of Science and Technology in Society. The results showed that after the didactic interventions, respondents were up to date with the effects of technology in the social world and believe that technological advancement can improve the quality of life when used with discernment.
\end{abstract}

Keywords: Science. Technology. Education. 


\section{Introdução}

\subsection{Problema/Questão}

A disciplina Ciência, Tecnologia e Sociedade (CTS) no Curso de Química da Universidade Estadual do Ceará é uma disciplina optativa, que começou a ser ofertada em 2013, e até o momento só é ministrada pela Profa ${ }^{a} r^{a}$ Micheline Soares Costa Oliveira. No início da disciplina verificou-se que os alunos nada sabiam da existência da relação CTS e não compreendiam os impactos que as duas primeiras impõem na sociedade não alfabetizada cientificamente. Observando a necessidade desse aprimoramento, foram elaboradas estratégias de ensino como forma de divulgação e conscientização dos discentes e até dos docentes sobre CTS.

\subsection{Revisão da Literatura}

Na contemporaneidade, a ciência e a tecnologia se encontram tão vigentes e inseridas no meio social, que raramente percebe-se a diferença entre ambas; e inclusive, a maioria da população leiga não consegue diferenciar os conceitos e muito menos entender a relação entre elas e a sociedade. Essa relação interfere em diversos ramos da coletividade como, por exemplo, a política, economia, cultura, e também, em problemas práticos do cotidiano (SANTOS; MORTIMER, 2002).

A tecnologia age nas forças produtivas utilizando os mais variados métodos, ferramentas, mecanismos e inteligências; $e$, um dos grandes objetivos é justamente organizar e perenizar os vínculos sociais. Deste modo, a crescente presença da ciência e da tecnologia nas atividades produtivas e nas relações sociais estabelece um ciclo permanente de mudanças remodelando o significado do trabalho no contexto da globalização. Como as questões da Natureza da Ciência ( $\mathrm{NdC}$ ) constituem hoje um dos elementos centrais e inovadores da alfabetização científica e tecnológica e, portanto, configuram-se como um aspecto essencial do currículo de ciências (ACEVEDO, VÁZQUEZ E MANASSERO, 2001); tornando-se, assim, necessário compreender as tênues relações entre Ciência, Tecnologia e Sociedade.

No contexto da sociedade científico-tecnológica, segundo Pinheiro, Silveira e Bazzo (2009), é imprescindível que o estudante tenha amplo domínio das ferramentas de estudo para que consiga, de maneira adequada, interpretar o mundo. As propostas de ensino com enfoque CTS, por sua vez, situam os conteúdos acadêmicos e escolares na conjuntura do seu meio tecnológico e social e permitem que os estudantes desenvolvam o pensamento crítico, e integrem o conhecimento científico com a tecnologia em suas experiências de vida.

Partindo da necessidade de aproximar os estudantes, e também os professores, do conhecimento de ciência e tecnologia, as instituições de ensino devem-se colocar como elemento central desta aproximação. A formação do cidadão que seja capaz de interagir com a dinâmica atual da sociedade é necessária para que o cidadão seja atuante na sociedade, de forma crítica e ativa no sentido de compreender o desenvolvimento tecnocientífico.

Um recurso amplamente utilizado que contribui para o desenvolver a capacidade de construção de argumentações é a controvérsia controlada, já que exige um leque de conhecimentos do aprendiz em relação aos seus pontos de vista para fundamentar suas argumentações; e ainda, fornecer as conexões necessárias para a compreensão do tema, exigindo um conjunto de capacidades imprescindíveis ao exercício da cidadania nos tempos atuais: a seleção e o tratamento da informação, a busca do consenso em meio a opiniões conflitantes e o discernimento necessário à tomada de decisões (REIS, 2013).

Considerando a assunção do caráter polissêmico e da não transparência da linguagem da perspectiva educacional CTS, a abordagem aqui realizada deve ser compreendida como inserida no âmbito do que se tem denominado de perspectiva CTS crítica (DAGNINO, 2008), que se aproxima e complementa a 
perspectiva educacional crítica no sentido Freireano. Não é uma abordagem que se possa fazer com facilidade no ensino de $C \& T$, principalmente se pensarmos na multiplicidade e complexidade que envolve a educação escolar, do ato de ensinar e de todos os demais aspectos que não têm sido contemplados pelo enfoque educacional CTS.

Para visualizar melhor o cenário de opiniões e ideias que os estudantes fazem dessas questões, serão analisadas as percepções que esses futuros professores têm do ensino com enfoque CTS verificando se as abordagens didáticas empreendidas por eles permitem a construção de aprendizagens significativas e contextualizadas em relação a questões sociais que envolvem Ciência e Tecnologia (SOLBES \& VILCHES, 1998)

Para análise utilizou-se o modelo pregado pelo Projeto Ibero-americano de Atitudes Relacionadas com Ciência, Tecnologia e Sociedade - PIEARCTS, que é uma investigação cooperativa internacional, desenvolvida por uma equipe de investigadores em diferentes lugares de 7 países ibero-americanos (Argentina, Brasil, Colômbia, Espanha, México, Panamá e Portugal). O objetivo central do PIEARCTS é o diagnóstico da compreensão da $\mathrm{NdCeT}$ em temas CTS, com mostras de estudantes e professores, para informar e identificar sobre seus pontos fortes e fracos, melhorar o ensino e a aprendizagem da ciência e da tecnologia (planejamento, elaboração e inovação do currículo), melhorar a formação inicial e continuada dos professores, comparar resultados e consolidar equipes de investigação nos países participantes. Este estudo pretende oferecer resultados numa perspectiva transnacional (VAZQUEZ et. al., 2011).

\subsection{Propósito}

Avaliar o resultado de intervenções didáticas na disciplina de Ciência, Tecnologia e Sociedade (CTS) no curso de Licenciatura em Química da Universidade Estadual do Ceará (UECE), utilizando como ferramenta de avaliação o questionário do PIEARCTS, para formação dos futuros professores.

\section{Metodologia}

Como a disciplina de CTS no Curso de Química da UECE está em construção, têm sido aprimoradas estratégias e novas metodologias para o melhor aproveitamento dessas informações pelos alunos. Assim iniciou-se o semestre com as aulas de conhecimento gerais sobre CTS e temas específicos como: Lixo Nuclear, Desastres Ambientais, Uso de aplicativos e Mídias Sociais, Tecnologia nas Escolas, Biotecnologia, Nanotecnologia, Tecnologias do futuro, Impactos sociais de CTS, entre outros.

A prática aplicada visou contribuir para formação dos futuros professores de Química utilizando os temas mencionados, para estimular e desafiar os alunos a conceberem seus próprios conceitos. A pesquisa fortificou-se na ação do planejamento da disciplina, ao serem realizadas aulas expositivas, seminários, apresentação de documentário, vídeos, palestras e jogos; inclusive após a construção de casos de experiências vivenciadas durante o processo ensino-aprendizagem. Essa busca de aprimoramento da disciplina criou uma nova perspectiva para aulas tradicionais, tornando as aulas mais discursivas e com mais participação dos alunos.

Ao final do curso foi aplicado o Questionário de Opiniões sobre a Ciência e a Tecnologia (COCTS) do Projeto Ibero-americano de Atitudes Relacionadas com Ciência, Tecnologia e Sociedade (PIEARCTS), destinado a avaliar as atitudes e opiniões em CTS (AZEVEDO et al., 2001), o qual é composto por 100 questões de múltipla escolha. O modelo escolhido para aplicação é uma versão baseada no original e idealizada pela equipe de CTE do Centro Federal de Educação Tecnológica Celso Suckow da Fonseca (CEFET - RJ) localizado na cidade do Rio de Janeiro. 
Questionário de Opiniões sobre a Ciência e a Tecnologia (COCTS) é baseado na avaliação das frases componentes das questões por 16 juízes peritos de reconhecida formação acadêmica nas diversas áreas da temática exposta. O instrumento utilizando pelos juízes para avaliação das frases é a Escala de Likert, a qual atribui valores de 1 a 9 pontos expressando em diferentes níveis o índice de concordância ou não com o conteúdo das frases. Para o grupo de respostas ingênuas, tem-se o valor 1 para totalmente ingênua, o valor 2 para muito ingênua e o valor 3 para ingênua. Já as respostas plausíveis são indicadas pelos seguintes valores: 4 para pouco plausível, 5 para plausível e 6 para muito plausível. Por fim, os valores 7, 8 e 9 representam, respectivamente, adequada, pouco adequada e muito adequada. (Silva, Antonioli, Chrispino, 2011 Apud Vázquez et al., 2005).

Após o respondente atribuir o grau de concordância, entre 1 e 9 , para cada uma das frases das questões, estes valores são convertidos em um intervalo de $[-1,+1]$ criando-se um índice atitudinal, que representa os indicadores quantitativos das opiniões e atitudes dos entrevistados sobre $\mathrm{NdC}$ com enfoque CTS. Se, por comparação com a classificação previamente feita pelos juízes, as respostas obtiverem índice igual ou maior que (+1), caracteriza-se uma atitude informada, crítica, contemporânea e contextualizada aos padrões desses juízes. No entanto, se índice for igual ou próximo a (-1), a opinião é considerada desinformada, simplista ou inexperiente. Os índices obtidos foram chamados de Índice Atitudinal (Ia), parâmetro usado para expressar os resultados obtidos compará-los a valores encontrados na literatura.

Tais questionamentos foram apresentados aos dezesseis alunos do Curso de Química da UECE matriculados na disciplina de CTS no semestre 2016.1; utilizando uma versão do formulário 1 (F1) que contém 10 questões sobre assuntos nas temáticas Ciência, Tecnologia e Sociedade. Destas, analisou-se prioritariamente as questões F1-20141, F1-20411, F1-40161, F1-40221 e F1-40531, com ênfase nas duas últimas.

\section{Resultados}

As frases das questões foram classificadas pelos juízes peritos do projeto PIEARCTS em: Ingênuas, Plausíveis e Adequadas; como visualizado na tabela 01.

\begin{tabular}{cccccc}
\multicolumn{7}{c}{ Tabela 01 - Classificação dos itens para as questões selecionadas } \\
\hline QUESTÕES/ITENS & $\mathbf{2 0 1 4 1}$ & $\mathbf{2 0 4 1 1}$ & $\mathbf{4 0 1 6 1}$ & $\mathbf{4 0 2 2 1}$ & $\mathbf{4 0 5 3 1}$ \\
\hline A & Adequada & Plausível & Ingênua & Plausível & Ingênua \\
B & Adequada & Plausível & Plausível & Adequada & Plausível \\
C & Adequada & Plausível & Adequada & Ingênua & Ingênua \\
D & Plausível & Plausível & Adequada & Ingênua & Adequada \\
E & Ingênua & Plausível & Plausível & Plausível & Adequada \\
F & Adequada & Ingênua & Adequada & Ingênua & Plausível \\
G & Plausível & Ingênua & - & - & - \\
H & Ingênua & - & - & - & - \\
I & Ingênua & - & - & - & - \\
J & Ingênua & - & - & - & - \\
\hline
\end{tabular}

A primeira questão deste formulário é a F1-20141 e, está incluída no âmbito da "Influência da Sociedade na C\&T", que pede a opinião do entrevistado sobre a interferência da política governamental no trabalho dos cientistas e o papel desses na sociedade. $O$ gráfico 01 apresenta a média geral dos índices atitudinais nessa questão, cujo valor é 0,284, um índice razoável segundo VÁZQUEZ, MAS \& TALAVERA (2010), ainda mais se considerar que dos 16 alunos, apenas dois obtiveram índice atitudinal abaixo de zero. É possível perceber que, em sua maioria, os entrevistados compreendem que o governo influencia na 
investigação científica, principalmente porque este último apoia as pesquisas e investigações por intermédio de financiamento e parcerias. Mas deve-se lembrar que os cientistas são partes integrantes e ativa da sociedade e estão sujeitos às políticas governamentais.

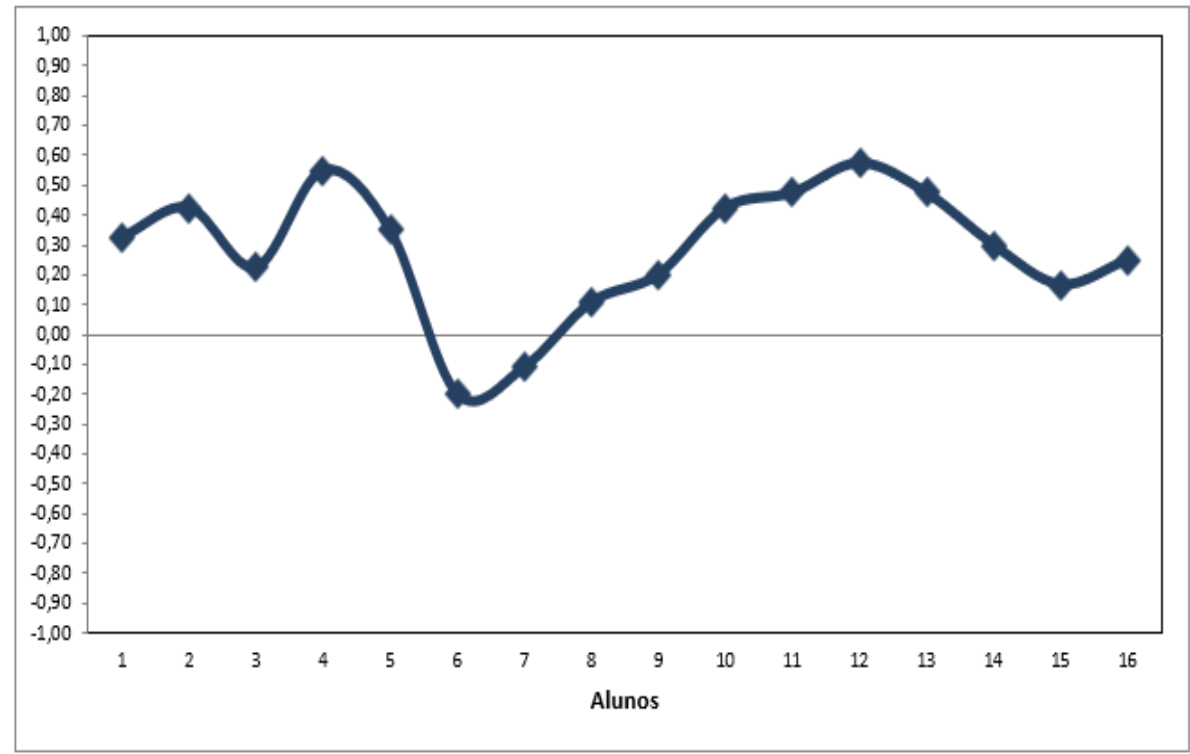

Gráfico 01 - Índice atitudinal dos respondestes, referente a questão 20141

A próxima questão, a F1-20411 ainda faz parte do âmbito "Influência da sociedade na C\&T"; mas fala sobre Ética, mais especificamente sobre a contribuição ou não da ética e da religião na investigação científica. Obteve média geral de -0,269, um índice baixíssimo visto que apenas 6 alunos dos 16 alcançaram índices atitudinais positivos para essa questão (gráfico 02 ).

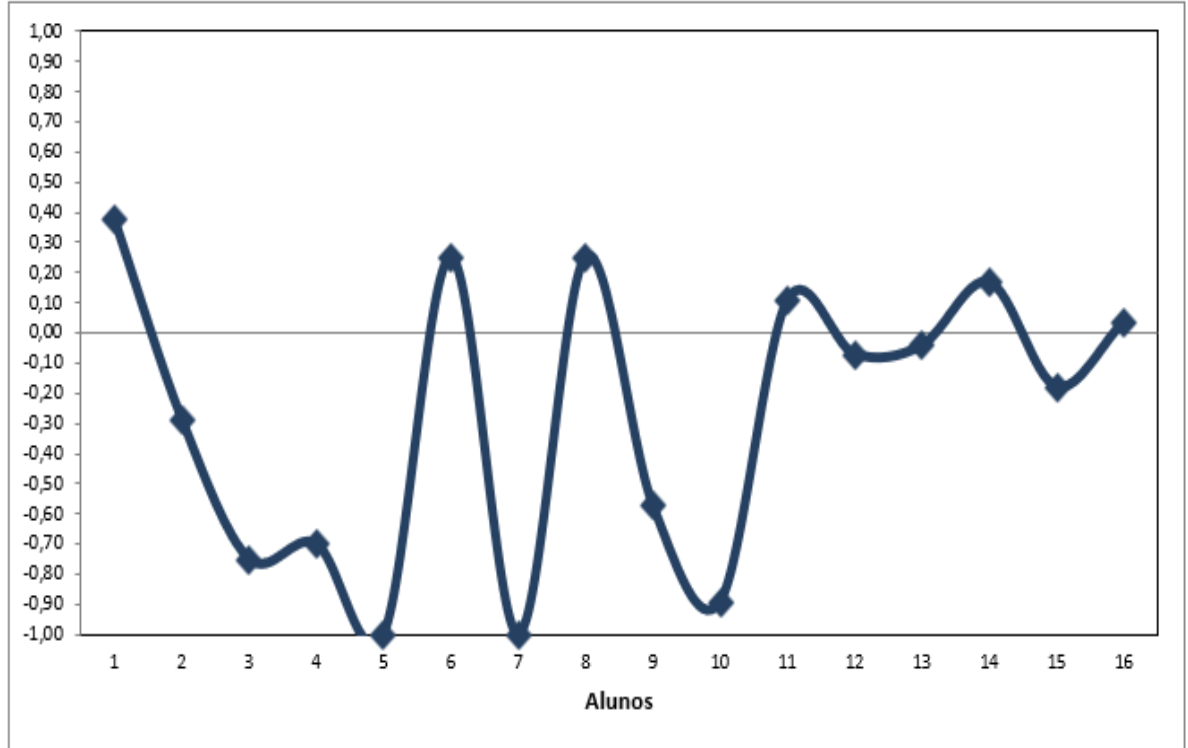

Gráfico 02 - Índice atitudinal dos respondestes, referente a questão 20411 
As questões F1-40161, F1-40221 e F1-40531 estão incluídas no tema "Influência da C\&T na Sociedade". A primeira vem falar da responsabilidade social, a segunda das decisões sociais e a terceira, do bem estar econômico, como visto no gráfico 03.

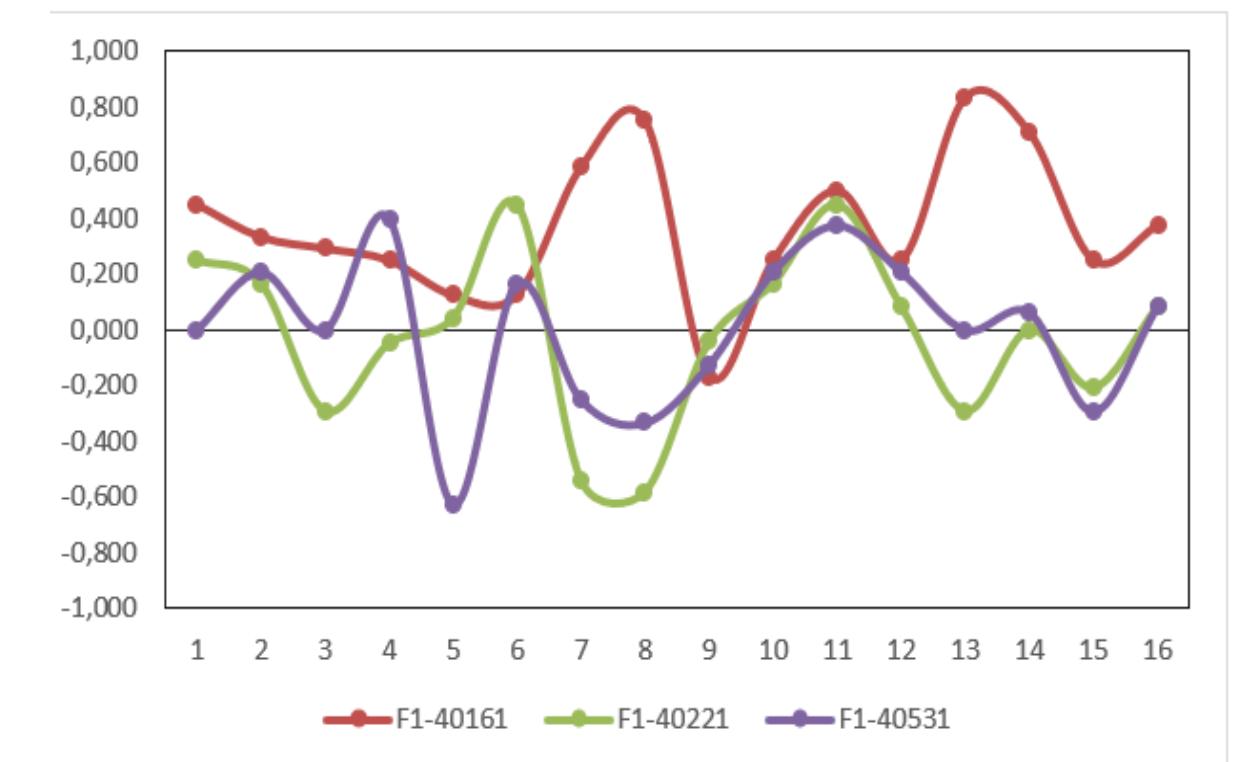

Gráfico 03 - Índice atitudinal dos respondestes, referente as questões F 1 - 40161, F1-40221 e F1-40531.

A questão F1-40161 obteve um Índice atitudinal (Ia) de 0,369, índice considerado bom e totalmente compatível com os encontrados com o projeto para o mesmo público alvo. Essa questão vem abordar temas como contaminação do meio ambiente e a responsabilidade dos "países desenvolvidos" e "não desenvolvidos" em produzirem e conterem essa contaminação. Pelo Ia, percebe-se que os respondentes têm uma postura contextualizada sobre o tema e conseguem definir o que melhor poderia ser feito, ou pelo menos ter uma ideia, do que poderia ser fazer de acordo com as proposições apresentadas.

Por suas respostas, os estudantes afirmam que reduzir ou eliminar a contaminação é o caminho mais viável para o lento processo de descontaminação. Contaminação essa, ocasionada pelas indústrias, incluindo a pesadas, desde que a Revolução Industrial instituiu um novo modo de vida, e pelo crescimento desordenado das grandes cidades. Os respondentes também percebem que transferir as indústrias pesadas para países considerados não desenvolvidos não ajuda muito nesse processo, podendo até piorar a situação propagando a contaminação em área antes limpas.

A F1-40221 foi perguntado como a Ciência e Tecnologia podem ajudar as pessoas a tomarem decisões morais. Essa questão é dividida em outras duas afirmações: 1. Ciência e Tecnologia podem ajudar nas decisões morais e 2. Ciência e Tecnologia não podem ajudar nas decisões morais. O índice atitudinal (Ia) é considerado baixo, $-0,020$, se comparado aos valores encontrados no projeto PIEARCTS, cujo valores variavam entre 0,05 a 0,3; e para os valores detectados por Silva (2012), que para essa questão obteve Ia de 0,192 . Observa-se, assim, que o conhecimento obtido no curso ainda não foi o suficiente para expandir os conceitos e fazer o estudante um ser ciente consciente e orientado sobre essa temática, revelando a debilidade e restrição do uso dos conceitos CTS.

Interpretando os dados obtidos no único item considerado Adequado dessa questão, conclui-se que os respondentes consideram que as decisões morais devem ser tomadas pelas pessoas e que a Ciência e Tecnologia ajudam a fornecer as informações básicas para que essas decisões sejam tomadas da melhor maneira possível, pois por si só C\&T não tomam decisões. O que é confirmado em outro item da mesma 
questão onde a maioria dos participantes acreditam que a C\&T tem a ver com essas decisões e que a ciência deve estar interligada e atuante na vida da população.

E na terceira pergunta (F1-40531) foi questionado se a tecnologia melhorará o nível de vida do país. Essa questão possui dois itens considerados Adequado, e obteve um Ia de 0,005. Quando comparado aos dados obtidos por Silva (2012) cujo Ia para essa questão foi de -0,123 e para o projeto PIEARCTS, entre 0,0 e $-0,4$; o Ia encontrado tem um leve aumento que pode caracterizar a desconstrução do pensamento de que o desenvolvimento se dá de modo linear.

Baseando-se nessa perspectiva, a maioria dos estudantes acreditam que a tecnologia pode melhorar a vida da população, mas também temem que o uso indiscriminado dessa ferramenta possa ocasionar prejuízos para toda a sociedade. Pode-se dizer então, que aquela visão linear dos conceitos de CTS: mais ciência equivale a mais tecnologia, que equivale a mais riqueza e consequentemente mais bem-estar social - está começando a ser desconstruída e surgindo uma nova maneira de ver as interações CTS, uma visão mais ampla, mais contextualizada e mais fiel à realidade.

O que chamou a atenção foi que os entrevistados consideram que a tecnologia ajuda a tornar a vida mais agradável, eficiente e divertida. Deve-se, aqui, levar em consideração a atual revolução tecnológica e sua intensa conectividade e a afirmação que os nascidos nessa era tecnológica têm em alta conta essa característica. Não têm influências antigas, pois só conhecem brincadeira e jogos dessa era, assim, podem lhes faltar uma visão mais crítica e contemporânea sobre o tema, capaz de avaliar os desafios e impactos que a ciência e tecnologia podem trazer para o futuro, de acordo com os índices apresentados na Tabela 02.

Tabela 02 - Médias dos Índices Atitudinais das questões apresentadas nesta pesquisa

\begin{tabular}{ccccccc}
\hline $\begin{array}{c}\text { ALUNO / } \\
\text { QUESTÕES }\end{array}$ & $\mathbf{F 1 ~ 2 0 1 4 1}$ & $\mathbf{F 1 ~ 2 0 4 1 1}$ & $\mathbf{F 1 ~ 4 0 1 6 1}$ & $\mathbf{F 1 ~ 4 0 2 2 1}$ & $\mathbf{F 1 ~ 4 0 5 3 1}$ & $\begin{array}{c}\text { MÉDIA / } \\
\text { ALUNO }\end{array}$ \\
\hline 1 & & & & & & \\
\hline & 0,325 & 0,375 & 0,450 & 0,250 & 0,000 & 0,280 \\
3 & 0,425 & $-0,286$ & 0,333 & 0,167 & 0,208 & 0,169 \\
4 & 0,225 & $-0,750$ & 0,292 & $-0,292$ & 0,000 & $-0,105$ \\
5 & 0,550 & $-0,700$ & 0,250 & $-0,050$ & 0,400 & 0,090 \\
6 & 0,350 & $-1,000$ & 0,125 & 0,042 & $-0,625$ & $-0,222$ \\
7 & $-0,200$ & 0,250 & 0,125 & 0,450 & 0,167 & 0,158 \\
8 & $-0,111$ & $-1,000$ & 0,583 & $-0,542$ & $-0,250$ & $-0,264$ \\
9 & 0,107 & 0,250 & 0,750 & $-0,583$ & $-0,333$ & 0,038 \\
10 & 0,200 & $-0,571$ & $-0,167$ & $-0,042$ & $-0,125$ & $-0,141$ \\
11 & 0,425 & $-0,893$ & 0,250 & 0,167 & 0,208 & 0,031 \\
12 & 0,475 & 0,107 & 0,500 & 0,450 & 0,375 & 0,381 \\
13 & 0,575 & $-0,071$ & 0,250 & 0,083 & 0,208 & 0,209 \\
14 & 0,475 & $-0,043$ & 0,833 & $-0,292$ & 0,000 & 0,195 \\
15 & 0,300 & 0,167 & 0,708 & 0,000 & 0,063 & 0,248 \\
16 & 0,167 & $-0,179$ & 0,250 & $-0,208$ & $-0,292$ & $-0,052$ \\
QÉDIA / & 0,250 & 0,036 & 0,375 & 0,083 & 0,083 & 0,165 \\
\hline & 0,284 & $-0,269$ & 0,369 & $-0,020$ & 0,005 & \\
\hline Q & & & & & & \\
\hline
\end{tabular}




\section{Discussão e conclusão}

Em relação à questão F1-20141, os respondentes exibem compreensão do âmbito de interferência do governo nas pesquisas científicas e conseguem perceber que este último não controla totalmente o que deve ser pesquisado, pois os cientistas têm determinadas atribuições, conhecimentos e capacidades peculiares ao estudo e ofício desempenhado, que não são pertinentes à alçada governamental. Esse resultado é condizente ao encontrado por Silva, Antonioli e Crispiano (2011) em pesquisa semelhante em uma unidade de ensino. Se comparar o valor obtido com os Índices atidutinais (Ias) encontrados no projeto PIEARCTS para os países participantes da pesquisa, o valor também é semelhante, considerado assim, aceitável.

Comparando os dados obtidos na questão F1-20411 com os de ROIG et al, (2010), quando da pesquisa nos sete países IberoAmericanos, podemos perceber que o Ia obtido pelos alunos da UECE seguem o mesmo padrão obtido pelo projeto. Inclusive entre os próprios professores de Ciências, esse é um índice que tende ao negativo, indicando que ética é uma temática ampla, diversificada e cheia de controvérsias; justamente porque uma pesquisa científica tem nuances tênues entre o que é ético e o que é amoral, pois pode ferir crenças, culturas, grupos sociais e direitos humanos.

As questões F1-40221 e F1-40531 comparada com a pesquisa realizada no Centro Federal de Educação Tecnológica do Rio de Janeiro (CEFET/RJ) entre os anos de 2010 e 2012 usando o mesmo conceito de respostas, notou-se que os alunos da UECE mantiveram um padrão aceitável equiparando-se em conceitos e atitudes cabíveis, significando que os princípios aprendidos em sala de aula têm contribuído para a formação do cidadão e a sua conscientização enquanto elemento transformador do meio (Tabela 03).

Tabela 03 - Comparação dos la obtidos nesse trabalho na UECE com os valores do CEFET-RJ.

\begin{tabular}{cccccc}
\hline $\mathbf{4 0 2 2 1}$ & UECE & CEFET - RJ & $\mathbf{4 0 5 3 1}$ & UECE & CEFET - RJ \\
\hline ITEM & Ia & Ia & ITEM & Ia & Ia \\
${ }^{*}$ A & $-0,1406$ & $-0,1324$ & A & $-0,3437$ & $-0,2157$ \\
B & 0,1462 & 0,5297 & B & $-0,2656$ & $-0,099$ \\
C & $-0,3906$ & $-0,1683$ & C & 0,1250 & $-0,3039$ \\
${ }^{*} D$ & 0,3593 & 0,4010 & ${ }^{*} D$ & 0,2031 & 0,2778 \\
${ }^{*} \mathrm{E}$ & $-0,0781$ & $-0,0971$ & $\mathrm{E}$ & 0,5937 & 0,4345 \\
$F$ & 0,2343 & 0,4436 & $\mathrm{~F}$ & 0,0312 & $-0,0343$ \\
\hline
\end{tabular}

*Valores semelhantes

As semelhanças de respostas foram principalmente nos itens das questões relacionadas com o a influência da tecnologia em suas decisões e melhoria de vida. Mesmo de realidades diferentes, esses jovens já estão formando uma consciência científica e poderão contribuir em muito no futuro para as próximas gerações.

Em análise ao Índice Atitudinal Global (Iag) da cada questão: Iag = 0,0234 para F-40221, e Iag = 0,0573 para F1-40531. Notou-se que os resultados estão dentro do intervalo de valores expressos pela metodologia Ibero-americana. Silva (2012), porém, encontrou valores diferentes para a mesma questão, credita-se que essa diferença foi devido à aplicação da pesquisa em um curso de Educação à distância.

Quase 70\% dos respondentes mantêm a crença de que a tecnologia sempre traz novidade e bonança e que assim será no futuro. Isso, ou contradiz o que os mesmos afirmaram anteriormente - que a tecnologia tem seu lado bom, mas que pode trazer malefícios se não for bem utilizada - ou os participantes mantêm a fé de que o ser humano em seu âmago é essencialmente bom e que mesmo 
com as diversas implicações negativas ele se manterá íntegro, resultado semelhante observados por Berk, da Matta \& Chrispino (2015), quando trataram da visão de professores do Ensino Médio sobre o mesmo tema.

Conclui-se que as tecnologias digitais estão presentes na sociedade atual e mundialmente difundidas, os educadores devem estar dispostos a incentivar a inclusão dos alunos na era cyber. No presente trabalho, verificou-se que os entrevistados acreditam que a Ciência e Tecnologia ajudam sim a tomar grandes decisões morais e que melhorará a qualidade de vida, porém com receio de que a tecnologia possa vir a trazer complicações. Percebeu-se também como a disciplina CTS pode influenciar e abrir a percepção dos alunos envolvidos, gerando um novo ânimo para continuar o trabalho que vem sendo desenvolvido na disciplina, esclarecendo questões cotidianas relacionadas à CTS. Observou-se que o processo ensino-aprendizagem foi mais construtivo na maneira que a pesquisa-ação foi desenvolvida para formação de licenciados. Nessa dinâmica pretende-se ampliar os horizontes de alunos e chamálos para discussão, pois esse tipo de trabalho deve ser contínuo e multiplicador.

\section{Referências bibliográficas}

Acevedo, J. A., Vázquez, A., \& Manassero, M. A. (2001). El movimiento Ciencia, Tecnología y Sociedad y la enseñanza de las ciencias. En: M. A., Manassero; A. Vázquez; J. A. Acevedo (Eds) Avaluació dels temes de ciència, tecnologia i societat. (pp. 14-32) Palma de Mallorca, ES: Conselleria d'Educació i Cultura del Govern de les Illes Ballears.

Berk, A., Matta, R., \& Chrispino, A. (2015). A investigação das visões de professores formados e em formação acerca das interações entre ciência, tecnologia e sociedade. Colloquium Humanarum, vol. 12, (Especial), 1053-1061. doi: 10.5747/ch.2015.v12.nesp.000723

Dagnino, R. (2008). Neutralidade da ciência e determinismo tecnológico: um debate sobre a tecnociência. Campinas, SP: Editora da UNICAMP.

Pinheiro, N. A. M., Silveira, R. M. C. F., \& Bazzo, W. A. (2009). O contexto científico-tecnológico e social acerca de uma abordagem crítico-reflexiva: perspectiva e enfoque. Revista Iberoamericana de Educación, 49 (1), 1-12. Recuperado de http://rieoei.org/2846.htm

Reis, P. (2013). Da discussão à ação sócio-política sobre controversias sócio-científicas: uma questão de cidadania. Ensino de Ciências e Tecnologia em Revista, 3(1), 1-10. Recuperado de http://repositorio.ul.pt/bitstream/10451/9577/3/DA\%20DISCUSS\%C3\%83O\%20\%C3\%80\%20A\%C3\%8 7\%C3\%830.pdf

Roig, A. B., García-Carmona, A., Alonso, Á. V., \& Mas, M. A. M. Análisis comparativo transnacional de la comprensión de la NdCyT entre los países participantes en el proyecto PIEARCTS. En A. B. Roig, A. V. Alonso, M. A. M. Mas, A. García-Carmona (Eds) Documento de Trabajo n口 05. (pp. 30-53). Madrid, ES: Centro de altos Estudios Universitarios de la OEI.

Santos, W. L. P. \& Mortimer, E. F. (2002) Tomada de decisão para ação social responsável no ensino de ciências. Ciência \& Educação, (7)1, 95-111. doi: 10.1590/S1516-73132001000100007

Silva, M. A. F. B. da (2012). O Conceito de Tecnologia a partir das pesquisas do PIEARCTS. (Tesis de doctorado inédita). Centro Federal de Educação Tecnológica Celso Suckow da Fonseca, Brasil. 
UTE. Revista de Ciències de l'Educació 2018 núm. 2. Pàg. 52-61
Ciência e Tecnologia na Sociedade Digital: Visão de alunos do Curso de Licenciatura em Química

Solbes, J. \& Vilches, A. (1998): Las interacciones CTS en los nuevos textos de la enseñanza secundaria. En: E. B. Hernández, A. J. de Pro (Eds.), Investigación e Innovación en la Enseñanza de las Ciencias (pp. 142-147). España: DM

Vázquez, Á. A.; Mas, M. A. M. \& Talavera, M. (2010) Actitudes y creencias sobre naturaleza de la ciencia y la tecnología en una muestra representativa de jóvenes estudiantes. Revista Electrónica de Enseñanza de las Ciencias, 9 (2), 333-352. Recuperado de http://reec.uvigo.es/volumenes/volumen9/ART3_Vol9_N2.pdf

Vázquez, A.A., Manassero, M. A. M., Acevedo, J. A. (2005). Quantitative analysis of complex multiplechoice items in Science Technology and Society: item scaling. Revista Electrónica de Investigación Educativa, 7(1), 1-29. Recuperado de https://redie.uabc.mx/redie/article/viewFile/116/200

Vázquez A.A., Alonso, A.; Maciel, M. D., Chrispino, A. \& Manassero, M. A. (2011) A compreensão dos temas de ciência, tecnologia e sociedade no Brasil: análise comparativa com outros países do PIEARCTS. En: W. L. P. Santos, D. Auler (Eds.). CTS e educação científica: desafios, tendências e resultados de pesquisas. (pp. 211-240). Brasília: Editora Universidade de Brasília. 